\title{
Temporal Change of the Aggregation Response in Triatoma infestans
}

\author{
Alicia Nieves Lorenzo Figueiras ${ }^{+}$, Claudio Ricardo Lazzari \\ Departamento de Ciencias Biológicas, Facultad de Ciencias Exactas y Naturales, Universidad de Buenos Aires, \\ Ciudad Universitaria, (C1428EHA) Buenos Aires, Argentina
}

Adults and larvae of Triatoma infestans spend daylight hours assembled in shaded places. An assembling factor has been demonstrated in the excrement of this species. We analysed different aspects of the dynamics of the response of bugs. Recently fed insects do not aggregate around faeces. They start to show a significant assembling response from the 8th hour after feeding onwards. Just deposited faeces do not evoke assembling, but a significant rejection instead. This reaction switches $3 \mathrm{~h}$ after deposition, when the faeces become attractive to the insects. The attractiveness of faeces persists for about 10 days and can be recovered after this time by rehydration. These findings are discussed in relation to the biological role of faeces and the dynamics of the use of refuges by $\mathrm{T}$. infestans.

Key words: Triatominae - Chagas - aggregation behaviour - temporal-change

Triatoma infestans (Klug) (Hemiptera: Reduviidae) is an haematophagous bug of the subfamily Triatominae, that is the main vector of Chagas disease in South America, because it transmits the flagellate Trypanosoma cruzi.

During the day, triatomine bugs can be found assembled in protected places. The aggregation behaviour of $T$. infestans is mediated by thigmotaxis and also by volatile compounds. Odours present in their own faeces (Schofield \& Patterson 1977, Ondarza et al. 1986, Lorenzo Figueiras et al. 1994), as well as "foot-prints" left behind by bugs walking on substrates (Lorenzo Figueiras \& Lazzari 1998) can induce assembling in $T$. infestans. Faecal deposits act as chemical landmarks helping bugs to find refuges (Lorenzo \& Lazzari 1996). Nevertheless, only dry faeces are effective in assembling larvae and adults around them (Lorenzo Figueiras et al. 1994, Lorenzo Figueiras 1997). In contrast, fresh faeces do not promote the aggregation behaviour of $T$. infestans and seem even to induce rejection behaviour (Lorenzo Figueiras et al. 1994, Lorenzo Figueiras

This work received financial support from the University of Buenos Aires, CONICET/Argentina, and the WHO/UNDP/World Bank Special Programme for Research and Training in Tropical Diseases.

${ }^{+}$Corresponding author. Fax: +54 11-4576. 3384. E-mail: aliciaf@bg.fcen.uba.ar

Received 12 January 2000

Accepted 17 April 2000
1997). Concerning the chemical nature of the signal, Taneja and Guerin (1997) reported that ammonia is released from wetted faecal-papers and that this compound attracts triatomines.

The response of the bugs to the assembly signal depends on their nutritional stage. Schofield and Patterson (1977) showed that recently fed larvae of $T$. infestans and Rhodnius prolixus are not attracted to faeces, although Ondarza et al. (1986) suggested that in T. mazzotti an aggregation signal is present, which could act on unfed females and larvae.

Taken together, all these findings suggest that the response of bugs changes with time. On the one hand, the activity of the faeces depends on the time elapsed since deposited, and on the other, the response of bugs depends on their postfeeding time. In order to understand the functional role of the faecal assembling signal it becomes relevant to explore the dynamic changes in action and response, as well the persistence of the signal in the environment.

The aim of this work is to analyse the following aspects of the aggregation response of $T$. infestans: (1) the variation of the bugs' response according to the time elapsed after feeding; (2) the response induced by faeces in the bugs at different time intervals since deposited; (3) the persistence of the assembling signal in the environment; and (4) the subsequent recovery of the biological activity of the faeces.

\section{MATERIALS AND METHODS}

Experimental insects were 3rd, 4th and 5th-instar larvae of $T$. infestans from our laboratory colony. Faeces were collected from larvae on fil- 
ter papers during one day $(2 \times 3 \mathrm{~cm})$, avoiding any physical contact with the bugs, in order to exclude the presence of cuticle components that also induce aggregation (Lorenzo Figueiras \& Lazzari 1998). Experiments were conducted in a circular glass arena, as described previously (Lorenzo Figueiras et al. 1994). Briefly, three rectangular pieces of filter paper were placed equidistant on the arena floor. One of them was contaminated with faeces (experimental), whilst the others remained clean, as controls. Bugs were carefully released in the centre of the arena and their distribution was recorded after $1 \mathrm{~h}$.

The effect of starvation on the bugs' response Two experimental series were performed, in which the period of starvation of experimental insects was varied. In the first, we tested the aggregation response to dry faeces (three days old) of 4th-instar larvae at $0,1,2,4,6,8,16$ or $18 \mathrm{~h}$ after feeding. In the second series, we tested the response of 5thinstar larvae at 10,15,20, 25 or 30 days following a blood meal.

The persistence of the signal - This experimental series tested the aggregation behaviour of starved 4th-instar larvae (30 days postecdysis) to faeces deposited at different times before the assays. Filter papers impregnated with faecal material with an age of $1,2,3 \mathrm{~h}$ and $8,10,12,14$ or 21 days post deposition were used.

Activity restores experiments - We tested whether old and dry inactive faeces are able to recover their ability to induce aggregation after rehydration. Admitting that the aggregation factor is water-soluble (Cruz-López \& Morgan 1995, Lorenzo Figueiras 1997), and that old dry faeces lost their biological action, the addition of water could allow the expression of the aggregation factor (Cruz-López \& Morgan 1995, Lorenzo Figueiras 1997).

To test this hypothesis, the faeces were collected on filter papers from larvae of $T$. infestans. These papers were kept at $28^{\circ} \mathrm{C}$ during 14 days. As showed by Lorenzo Figueiras (1997), this period of time is sufficient to inactivate faeces. Thereafter, the treated papers were put into a glass bowl where they were immersed in $5 \mathrm{ml}$ of distilled water and stirred for $10 \mathrm{~min}$. The papers were then dried and tested for their ability to induce aggregation on the following day. Starved 3rd-instar larvae, seven days after ecdysis, were used as experimental insects. As before, one impregnated paper was tested against two clean control papers.

Control assays were made using papers contaminated with faeces, which were kept at $28^{\circ} \mathrm{C}$ during 14 days, but without rehydrating them, tested against two clean ones.
Statistical analysis - For each experimental series the distribution of the insects in the arena was statistically analysed by means of a G-test for goodness of fit to a random distribution (i.e., $1 / 3$ for experimental zone and $2 / 3$ for control zones). An ANOVA was used to compare the intensity of the aggregation response among assays. In this analysis, the data were transformed using the arcsine of the square root of the proportion and checked for normality and homogeneity of variances.

\section{RESULTS}

The effect of starvation on the bugs' response In the 1st experimental series, bugs were distributed at random between the experimental and the control papers, when tested at $0,1,2,4$ or $6 \mathrm{~h}$ after feeding (G-test, NS) (Fig. 1). However, when insects were tested at eight hours or more after the blood meal, they showed a significant tendency to aggregate on the impregnated paper (G-test, $P<$ 0.001) (Fig. 1). When we analysed whether the intensity of the response varied among assays, it proved to be the same at the different times (ANOVA, $\mathrm{F}=0.426 ; \mathrm{df}=23,2 ; P>0.05$ ).

In the 2 nd series of assays, assembly in the presence of dry faeces remained as a significant response of the bugs, independent of the time of starvation, and could be observed 30 days after feeding (G-test, $P<0.025$ ) (Fig. 1). The intensity of aggregation response did not differ with time elapsed since feeding $(\mathrm{F}=0.9123 ; \mathrm{df}=4,54 ; P>$ $0.05)$.

The persistence of the signal - The 3rd experimental series revealed that larvae distributed ran-

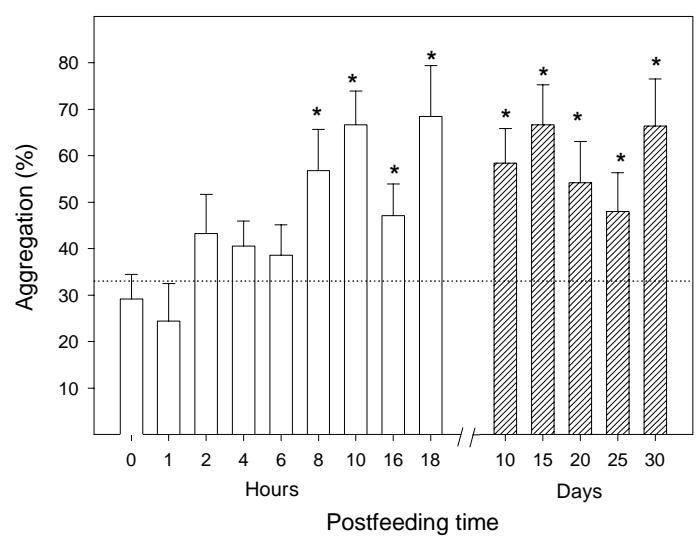

Fig. 1: the aggregation response of larvae of Triatoma infestans to dry faeces at different times after feeding. Asterisks indicate statistically significant differences. The horizontal line indicates the value expected from a random distribution. 
domly among control papers and experimental ones, when the later were impregnated with faeces deposed 1 or $2 \mathrm{~h}$ or more than 12 days before the assay (G-test, $P>0.05$ ) (Fig. 2). On the contrary, faeces having a postdeposition time ranging from $3 \mathrm{~h}$ to 12 days induced a significant aggregation of bugs on the impregnated papers, as compared with the control ones (G-test, $P<0.05$ ) (Fig. 2).

Activity restores experiments - Rehydration of papers impregnated with faeces which had lost their activity (i.e. older than 12 days), recovered their ability to induce a significant assembling response in the bugs (G-test, $P<0.001$, Fig. 3).

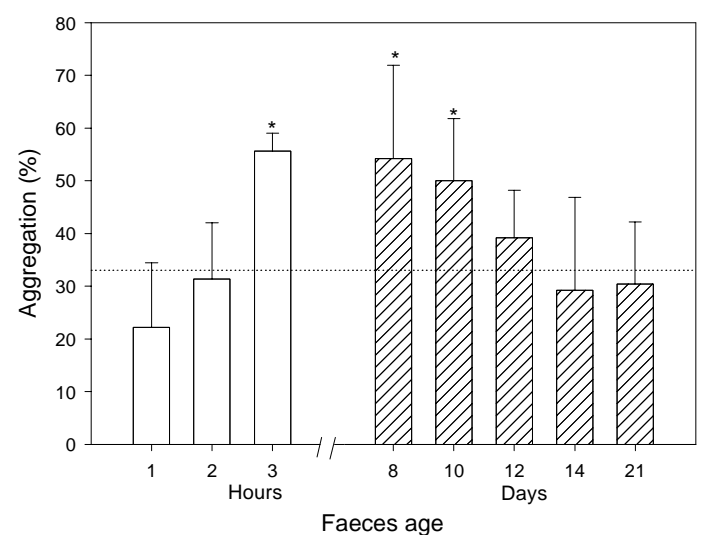

Fig. 2: the aggregation response of larvae of Triatoma infestans to faeces at different times after their deposition. The horizontal line indicates the value expected from a random distribution. Asterisks indicate aged faecal deposits that elicit significant assembling responses.

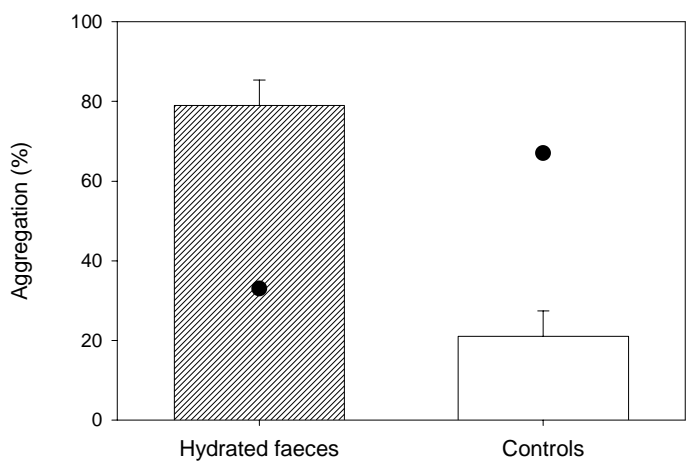

Fig. 3: the aggregation response of larvae of Triatoma infestans to a filter paper impregnated with rehydrated inactive faeces and to two control filter papers. The black points indicate the values expected from a random distribution (33\% for the experimental sector and $67 \%$ for the controls sectors). The insects significantly preferred the treated paper $(P<0.001)$.

\section{DISCUSSION}

Previous reports have suggested that the faecal assembling signal may act as a cue for orientation to potential refuges (Lorenzo \& Lazzari 1996, Lorenzo 1997). However, the lack of response of recently fed insects appeared to contradict this hypothesis, assuming that bugs should look for a protected place immediately after feeding. Our present results, together with the previous ones on the temporal organization of the bugs' behaviour, allow us to show that the contradiction is only apparent.

The circadian rhythm of spontaneous activity of $T$. infestans is split in two main endogenously controlled components, one burst of activity at dusk and a second one at dawn (Lazzari 1992). As shown by Lorenzo and Lazzari (1998) these activity periods are related to the search for food and for refuge, respectively. In addition, the bugs leave the refuges during the first hours of the night, while the entrance activity is performed at the end of the scotophase. Taking into account the bimodal pattern of spontaneous circadian activity, as well the dynamics of the movements into and out of the artificial refuges, it can be concluded that the bugs search for food at dusk and for refuges at dawn. Long-term monitoring of the nocturnal activity of T. infestans (Lorenzo 1997) showed that recently fed insects remain outside the refuges until dawn, performing a copious diuresis. Results presented here reveal the occurrence of a switch in the assembling response of the bugs taking place about 8-10 $\mathrm{h}$ after the blood meal. As a consequence, we are able to postulate that recently fed insects respond to assembling factors at the time to search for refuge, i.e. at dawn, but not immediately after feeding.

In addition, bugs do not respond to recently deposited faeces, but do respond to faeces deposited at least $3 \mathrm{~h}$ ago. Moreover, present and previous results by Lorenzo Figueiras et al. (1994) show that fresh faeces elicit a significant rejection. Up to now, we do not know what kind of transformation takes place in the excrement during this time. Either the active compound/s could be produced or some substance/s masking the attractant factor could vanish.

The attractiveness of faeces persists for about ten days after deposition and decays thereafter. As a consequence, in order to sustain their activity as chemical landmarks of refuges (Lorenzo \& Lazzari 1996) the mark should be renewed as long as the refuge is still in use by the bugs. When bugs no longer exploit a refuge, that place looses its particular significance. When the inactive faeces are rehydrated, however, they are able to recover their 
capacity to attract and assemble bugs. So, the effectiveness of the mark can be extended over time and reinforced by means of new depositions.

Taneja and Guerin (1997) showed that the ammonia is released after soaking papers with faecal deposits in distilled water for several hours. In addition, these authors reported that this compound attracts the bug $T$. infestans on a locomotion compensator. Therefore, the recovery of the biological action of faeces after rehydrating them could be due to the increase in the release of ammonia and/or other substances, after the interaction of faeces with water. These results have relevant implications for: (1) the recognition of the chemical nature of the active component/s; (2) for the use of insectary-collected faecal papers as a source of natural attractants for triatomines; (3) for the potential application of this attractant in the field, as a control tool; and (4) to comprehend the chemical ecology of Chagas disease vectors.

\section{ACKNOWLEDGEMENTS}

To the staff members of the Laboratory of Insect Physiology for many fruitful discussions, and to $\mathrm{M}$ Giurfa for correcting the manuscript.

\section{REFERENCES}

Cruz-López L, Morgan D 1995. Chemical investigation of aggregation behaviour of Triatoma bugs (Hemiptera: Reduviidae). J Chem Ecol 21: 20692078.

Lazzari CR 1992. Circadian organization of locomotion activity in the haematophagous bug Triatoma infestans. J Insect Physiol 38: 895-903.

Lorenzo Figueiras AN 1997. Comportamiento de
Agregación y Comunicación Intra- e Interespecífica en Triatominae, $\mathrm{PhD}$ Thesis, Universidad de Buenos Aires, $167 \mathrm{pp}$.

Lorenzo Figueiras AN, Lazzari CR 1998. Aggregation in the haematophagous bug Triatoma infestans: a novel assembling factor. Physiol Entomol 23: 3337.

Lorenzo Figueiras AN, Kenigsten A, Lazzari CR 1994. Aggregation in haematophagous bug Triatoma infestans: chemical signals and temporal pattern. $J$ Insect Physiol 40: 312-316.

Lorenzo MG 1997. Factores que Afectan la Distribución Espacial de la Vinchuca Triatoma infestans y Búsqueda de Nuevas Herramientas para su Control, PhD Thesis, Universidad de Buenos Aires, 117 $\mathrm{pp}$.

Lorenzo MG, Lazzari CR 1996. The spatial pattern of defecation in Triatoma infestans and the role of faeces as a chemical mark of the refuge. $J$ Insect Physiol 42: 903-907.

Lorenzo MG, Lazzari CR 1998. Activity pattern in relation to refuge exploitation and feeding in Triatoma infestans (Hemiptera: Reduviidae). Acta Trop 70: 163-170.

Ondarza RN, Gutiérrez-Martínez A, Malo EA 1986. Evidence for the presence of sex and aggregation pheromones from Triatoma mazzottii (Hemiptera: Reduviidae). J Econ Entomol 79: 688-692.

Schofield CJ, Patterson JW 1977. Assembly pheromone of Triatoma infestans and Rhodnius prolixus nymphs (Hemiptera: Reduviidae). J Med Entomol 13: 727734.

Taneja J, Guerin PM 1997. Ammonia attracts the haematophagous bug Triatoma infestans: behavioural and neurophisiological data on nymphs. J Comp Physiol A 181: 21-34. 